\title{
THE DIXMIER-DOUADY CLASS OF GROUPOID CROSSED PRODUCTS
}

\author{
PAUL S. MUHLY and DANA P. WILLIAMS
}

(Received 21 August 2002; revised 20 February 2003)

Communicated by G. Willis

\begin{abstract}
We give a formula for the Dixmier-Douady class of a continuous-trace groupoid crossed product that arises from an action of a locally trivial, proper, principal groupoid on a bundle of elementary $C^{*}$-algebras that satisfies Fell's condition.
\end{abstract}

2000 Mathematics subject classification: primary 46L05, 46L55.

\section{Introduction}

Throughout this note $G$ will denote a second countable, locally compact, principal groupoid with Haar system, $\mathscr{A}$ will denote an elementary $C^{*}$-bundle over $G^{(0)}$ that satisfies Fell's condition, and $\alpha$ will denote a continuous action of $G$ on $\mathscr{A}$ via isomorphisms. Thus the pair $(\mathscr{A}, \alpha)$ is exactly what is needed to define an element in the Brauer group $\operatorname{Br}(G)$ as defined in [4, Definition 2.14]. As a special case of [3, Theorem 1], it follows that the groupoid crossed product $C^{*}(G ; \mathscr{A})$ has continuoustrace if and only if $G$ is a proper principal groupoid. Thus if $C^{*}(G ; \mathscr{A})$ has continuous trace we can, and do, assume that that $G=\Omega{ }_{p}^{*} \Omega=\left\{\left(\omega, \omega^{\prime}\right) \in \Omega \times \Omega: p(\omega)=\right.$ $\left.p\left(\omega^{\prime}\right)\right\}$ for a continuous open surjection $p: \Omega \rightarrow Y$. In this case we say that $G$ is a locally trivial, proper principal groupoid. In this note, we want to consider the Dixmier-Douady class $\delta\left(C^{*}(G ; \mathscr{A})\right)$, and we compute $\delta\left(C^{*}(G ; \mathscr{A})\right)$ when $p$ is locally trivial. Our approach is motivated in part by [6, Section 1] where Raeburn and Rosenberg consider the case where $G$ is the transformation group groupoid $G=H \times \Omega$ with $\Omega$ a locally trivial principal $H$-bundle, and $\alpha$ is pulled back from a locally

(C) 2004 Australian Mathematical Society $1446-7887 / 04 \$ A 2.00+0.00$ 
unitary action $\gamma$ of $H$ on a stable continuous-trace $C^{*}$-algebra $B$. Just as in [6, Theorem 1.1], we show that we can assume that $\mathscr{A}$ is the pull-back $p^{*} \mathscr{B}$ by the orbit map $p: G \rightarrow G^{(0)} / G$ of some locally trivial $K$-bundle over $G^{(0)} / G$. (Throughout, $K$ will denote the space of compact operators on a separable, infinite dimensional Hilbert space. By a locally trivial $K$-bundle over a space $X$, we shall mean a locally trivial fibre bundle over $X$, with fibre $K$ and structure group $\operatorname{Aut}(K)$. We shall use [8] as our basic reference on fibre bundles, sheaves, cohomology, etc.) In the event $\alpha$ satisfies an additional hypothesis - similar to being pulled back from a locally unitary action as in [6] - we show that $\delta\left(C^{*}(G ; \mathscr{A})\right)$ is a naturally defined perturbation of $\delta(B)$ in complete analogy with $[6$, Theorem 1.5$]$.

\section{Locally trivial proper principal groupoids}

Suppose that $\Omega$ and $Y$ are second countable locally compact Hausdorff spaces, and that $p: \Omega \rightarrow Y$ is a continuous, open surjection. Let $G$ be the proper principal groupoid $\Omega * \Omega$ and identify $G^{(0)}$ with $\Omega$ and $G^{(0)} / G$ with $Y$. We'll say that $G$ is locally trivial if $p: \Omega \rightarrow Y$ is a locally trivial fibre bundle with fibre $X$. That is, we assume there is a cover $\mathbf{U}=\left\{U_{i}\right\}$ of $Y$ and continuous maps $h_{i}: p^{-1}\left(U_{i}\right) \rightarrow U_{i} \times X$ such that the diagram

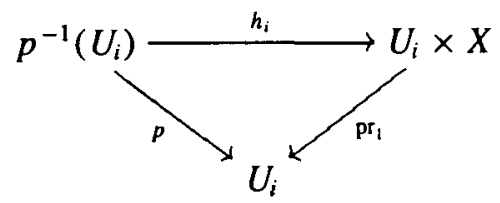

commutes for each $i$. In particular, we assume that there are continuous functions $\xi_{i}: p^{-1}\left(U_{i}\right) \rightarrow X$ such that

$$
h_{i}(w)=\left(p(\omega), \xi_{i}(\omega)\right) \text { for all } \omega \in p^{-1}\left(U_{i}\right)
$$

Consequently, if $U_{i j}:=U_{i} \cap U_{j} \neq \emptyset$, then the diagram

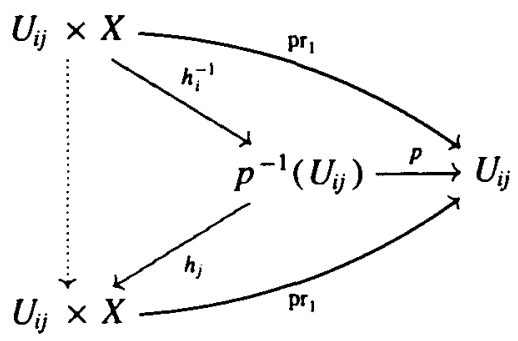


commutes. This, in turn, implies that for each $u \in U_{i j}$ there must be a continuous map $\phi_{i j}(u): X \rightarrow X$ such that

$$
h_{j} \circ h_{i}^{-1}(u, x)=\left(u, \phi_{i j}(u)(x)\right) \text { for all }(u, x) \in U_{i j} \times X .
$$

Further, since $\phi_{i j}(u)^{-1}=\phi_{j i}(u)$, we see that each $\phi_{i j}(u)$ is a homeomorphism of $X$. Consequently, $\phi_{i j}$ may be viewed as a function from $U_{i j}$ to $\operatorname{Homeo}(X)$. A straightforward computation shows that

$$
\phi_{i k}(u)=\phi_{j k}(u) \circ \phi_{i j}(u) \quad \text { for all } u \in U_{i j k}:=U_{i} \cap U_{j} \cap U_{k}
$$

REMARK 1. Homeo $(X)$ can be made into a topological group in such a way that $h_{n} \rightarrow h$ in Homeo $(X)$ if and only if given a net $x_{n} \rightarrow x$ in $X$ (with the same index set), then $h_{n}\left(x_{n}\right) \rightarrow h(x)$ and $h_{n}^{-1}\left(x_{n}\right) \rightarrow h^{-1}(x)$. Then it is not hard to see that $\phi_{i j}$ is continuous and that the transition functions $\left\{\phi_{i j}\right\}$ determine $p$ in the usual way.

EXAMPLE 2. Of course, the basic example of a locally trivial proper principal groupoid is the transformation groupoid associated to a principal (left) $H$-space $\Omega$ for a locally compact group $H$. In this case, the fibre space $X$ is just $H$, and we also want the local trivializations $h_{i}$ to be $H$-equivariant. In particular, there are continuous functions $s_{i j}: U_{i j} \rightarrow H$ such that $\phi_{i j}(u)(t)=t s_{i j}(u)$ for all $t \in H$. Furthermore, equation (3) is equivalent to

$$
s_{i j}(u) s_{j k}(u)=s_{i k}(u) \quad \text { for all } u \in U_{i j k} .
$$

Therefore the elements $\left\{s_{i j}\right\}$ determine the class $[p]$ of the principal bundle $p$ in $H^{1}(Y, \mathcal{H})$ where $\mathcal{H}$ is the sheaf of continuous $H$-valued functions and $H^{1}(Y, \mathcal{H})$ is the the first sheaf cohomology set determined by $\mathcal{H}[8$, Remark 4.54]. When we return to this example in the sequel, we will identify the transformation group groupoid $H \times \Omega$ with $G=\Omega \underset{p}{*} \Omega$ via the map $(t, \omega) \mapsto\left(\omega, t^{-1} \cdot \omega\right)$. (Recall that $H \times \Omega$ is the groupoid with unit space $\{e\} \times \Omega$ identified with $\Omega$ and with range and source maps $s(t, \omega)=t^{-1} \cdot \omega$ and $r(t, \omega)=\omega$. Then we have $(t, \omega)\left(s, t^{-1} \cdot \omega\right)=(t s, \omega)$ and $(t, \omega)^{-1}=\left(t^{-1}, t^{-1} \cdot \omega\right)$.)

REMARK 3. It is a matter of taste as to whether $\phi_{i j}$ or $\phi_{j i}$ appears in (2). Our taste might seem off in view of (3), but we have purposely endured bitter herbs in order enjoy (4) even if $H$ is not abelian. In either case, it is important fact of life that any formula for the Dixmier-Douady class that depends on standard topological data, such as transition functions like the $\phi_{i j}$, depends up to a sign on choices such as that made in (2). This will be important in comparing our result to other calculations in the literature (see Example 14). 
Now we want to see how the locally triviality of $p$ is reflected in the groupoid structure of $G$. We can define a topological groupoid isomorphism $k_{i}:\left.G\right|_{p^{-1}\left(U_{i}\right)} \rightarrow$ $X \times U_{i} \times X$ by

$$
k_{i}\left(\omega, \omega^{\prime}\right):=\left(\xi_{i}(\omega), p(\omega), \xi_{i}\left(\omega^{\prime}\right)\right)
$$

Here $X \times U_{i} \times X$ is the groupoid that has unit space identified with $X \times U_{i}$, orbit space identified with $U_{i}$ and multiplication given by $(x, u, y)(y, u, z)=(x, u, z)$. If $p^{(2)}: G \rightarrow G^{(0)} / G \cong Y$ is given by $p^{(2)}\left(\omega, \omega^{\prime}\right):=p(\omega)$, then we have commutative diagrams

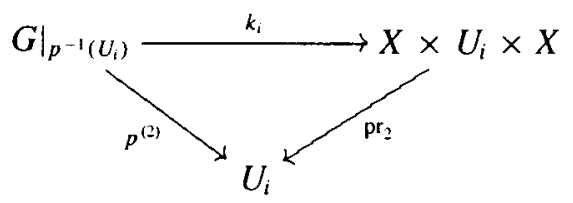

and

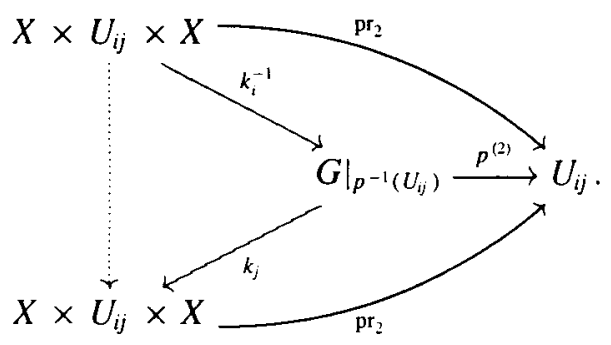

In particular, we claim that

$$
k_{j} \circ k_{i}^{-1}(x, u, y)=\left(\phi_{i j}(u)(x), u, \phi_{i j}(u)(y)\right) .
$$

To see this, consider

$$
\begin{aligned}
k_{j} \circ k_{i}^{-1}(x, u, y) & =k_{j}\left(h_{i}^{-1}(u, x), h_{i}^{-1}(u, y)\right) \\
& =\left(\xi_{j}\left(h_{i}^{-1}(u, x)\right), u, \xi_{j}\left(h_{i}^{-1}(u, y)\right)\right)
\end{aligned}
$$

which equals the right-hand side of (6) in view of (1) and (2).

We include the following lemma to motivate some of the constructions in the next section. At this point, it will be convenient to introduce the notation $\dot{\omega}$ for $p(\omega)$. This will make some of the more complicated formulas below, and elsewhere, a bit easier to digest.

LEMMA 4. Suppose that $p: \Omega \rightarrow Y$ is a locally trivial fibre bundle with fibre $X$ and that $G=\Omega \underset{p}{*} \Omega$. If $H$ is a topological groupoid and if $\alpha: G \rightarrow H$ is a continuous 
groupoid homomorphism, then there is open cover $\left\{U_{i}\right\}$ of $Y$ and continuous maps $\psi_{i}: p^{-1}\left(U_{i}\right) \rightarrow H$ such that

$$
\alpha\left(\omega, \omega^{\prime}\right)=\psi_{i}(w) \psi_{i}\left(\omega^{\prime}\right)^{-1} \quad \text { for }\left.\left(\omega, \omega^{\prime}\right) \in G\right|_{p^{-1}\left(U_{i}\right)} .
$$

REMARK 5. It is implicit in the statement of Lemma 4 that $r\left(\psi_{i}(\omega)\right)=r\left(\alpha\left(\omega, \omega^{\prime}\right)\right)$, while $s\left(\psi_{i}(\omega)\right)$ and, more generally, $\psi_{i}(\omega)^{-1} \psi_{j}(\omega)$ depend only on $p(\omega)$, for $\omega \in p^{-1}\left(U_{i j}\right)$.

Proof of Lemma 4. Choose $\left\{U_{i}\right\}, h_{i}: p^{-1}\left(U_{i}\right) \rightarrow U_{i} \times X$ and $k_{i}:\left.G\right|_{p^{-1}\left(U_{i}\right)} \rightarrow$ $X \times U_{i} \times X$ as above. For each $i$, fix $z_{i} \in X$ and define

$$
\psi_{i}(\omega):=\alpha \circ k_{i}^{-1}\left(\xi_{i}(\omega), \dot{\omega}, z_{i}\right)
$$

and observe that if $\left.\left(\omega, \omega^{\prime}\right) \in G\right|_{p^{-1}\left(U_{t}\right)}$, then

$$
\begin{aligned}
\alpha\left(\omega, \omega^{\prime}\right) & :=\alpha \circ k_{i}^{-1}\left(\xi_{i}(\omega), \dot{\omega}, \xi_{i}\left(\omega^{\prime}\right)\right) \\
& =\alpha \circ k_{i}^{-1}\left(\xi_{i}(\omega), \dot{\omega}, z_{i}\right) \alpha \circ k_{i}^{-1}\left(z_{i}, \dot{\omega}, \xi_{i}\left(\omega^{\prime}\right)\right)=\psi_{i}(\omega) \psi_{i}\left(\omega^{\prime}\right)^{-1}
\end{aligned}
$$

as required.

\section{The Dixmier-Douady class}

First, it will be helpful to recast [3, Theorem 1] in terms of Brauer groups as defined in [4]. (We follow the notation and terminology of [4]. In particular, $\mathfrak{B r}(G)$ denotes the collection of pairs $(\mathscr{A}, \alpha)$ of the kind we have been considering, while $\operatorname{Br}(G)$ denotes their Morita equivalence classes.) To start off, we only need $G$ to be a proper and not necessarily locally trivial. Our next result should be compared with [4, Proposition 11.2].

THEOREM 6. If $G$ is a second countable, locally compact, proper principal groupoid, then there is an isomorphism of $\operatorname{Br}(G)$ onto $\operatorname{Br}\left(G^{(0)} / G\right)$ which sends $(\mathscr{A}, \alpha) \in$ $\operatorname{Br}(G)$ to the class of the bundle representing the crossed product $C^{*}(G ; \mathscr{A})$ in $\operatorname{Br}\left(G^{(0)} / G\right)$. The inverse is given by sending $\mathscr{B} \in \mathfrak{B r}\left(G^{(0)} / G\right)$ to $\left(p^{*} \mathscr{B}, \tau \otimes 1\right)$ where $p: G^{(0)} \rightarrow G^{(0)} / G$ is the quotient map, $p^{*} \mathscr{B}=\left\{(\omega, b): \dot{\omega}=p_{\mathscr{B}}(b)\right\}$ is the pull-back and $(\tau \otimes 1)_{\left(\omega, \omega^{\prime}\right)}\left(\omega^{\prime}, b\right)=(\omega, b)$.

ProOF. It follows from [5, Proposition 2.2] and [4, Theorem 4.1] that $[\mathscr{A}, \alpha] \mapsto$ $\left[\mathscr{A}^{G^{(i)}}\right]$ is an isomorphism of $\operatorname{Br}(G)$ onto $\operatorname{Br}\left(G^{(0)} / G\right)$, where $\mathscr{A}^{C^{(3)}}=s_{*}(\mathscr{A}) / G$ and where $s_{*}(\mathscr{A})$ is the pull-back of $\mathscr{A}$ to $G$ via the source map $s$ (see, for example, [4, 
page 914]). Furthermore, [3, Theorem 1] implies that $C_{0}\left(G^{(0)} / G, \mathscr{A}^{G^{(0)}}\right)$ is Morita equivalent to $C^{*}(G ; \mathscr{A})$. This proves the first assertion.

On the other hand, [4, Theorem 4.1] implies that the inverse is given by

$$
[\mathscr{B}] \mapsto\left[\mathscr{B}^{G^{(0) \rho p}}, \text { id }^{G^{(0) \text { p }}}\right],
$$

where $G^{(0) \text { op }}$ is $G^{(0)}$ regarded as a left- $G$, right- $G^{(0)} / G$ equivalence (see, for example, [4, page 924]). However, $G^{\left(0^{\circ p}\right.}$ is easily seen to be isomorphic (as an equivalence) to the graph of $p, \operatorname{Gr}(p)$, where $\operatorname{Gr}(p):=\left\{(\omega, \dot{\omega}): \omega \in G^{(0)}\right\}$ (see also Section 6 of [4]). Therefore [4, Lemma 6.5] implies that

$$
\left[\mathscr{B}^{G^{(1) / p}}, \mathrm{id}^{G^{(0) / p}}\right]=\left[\mathscr{B}^{\operatorname{Gr}(p)}, \mathrm{id}^{\operatorname{Gr}(p)}\right]=\left[p^{*} \mathscr{B}, p^{*} \mathrm{id}\right] .
$$

Noting that $p^{*}$ id $=\tau \otimes 1$ completes the proof.

As a corollary, we get the following version of [6, Theorem 1.1].

COROLlary 7. Suppose that $[\mathscr{A}, \alpha] \in \operatorname{Br}(G)$. Then there is a locally trivial $K$ bundle $\mathscr{B}$ over $Y$ such that $[\mathscr{A}, \alpha]=\left[p^{*} \mathscr{B}, \beta\right]$. In particular, the Dixmier-Douady class $\delta(\mathscr{A})$ must lie in the image $p^{*}\left(H^{3}(Y ; \mathbb{Z})\right)$ of the map $p^{*}: H^{3}(Y ; \mathbb{Z}) \rightarrow H^{3}(\Omega ; \mathbb{Z})$ induced by $p$.

PROOF. Since $\tau_{G^{(0)}}:=\left(G^{(0)} \times K, \tau \otimes 1\right)$ acts as the identity in $\operatorname{Br}(G)$, we can replace $(\mathscr{A}, \alpha)$ with $\left(\mathscr{A} \otimes{G^{(i)}}\left(G^{(0)} \times K\right), \alpha \otimes(\tau \otimes 1)\right)$. Thus we may as well assume that $A:=C_{0}\left(G^{(0)}, \mathscr{A}\right)$ is stable. It follows from Theorem 6 and [7, Proposition 1.4(1)] that $\delta(\mathscr{A}):=\delta(A) \in p^{*}\left(H^{3}(Y ; \mathbb{Z})\right)$. Thus there is a stable continuous-trace $C^{*}$ algebra $B$ with spectrum $Y$ such that $A=p^{*} B$ [7, Proposition 1.4(2)]. In view of [8, Proposition 5.59], we may assume that $B=C_{0}(Y, \mathscr{B})$ where $\mathscr{B}$ is a locally trivial $K$-bundle with structure group Aut $K$ as required.

Let $\mathscr{B}$ be a locally trivial $K$-bundle over $Y$ as in Corollary 7 . It follows from [8, Proposition 4.53] for example, that there is a cover $\mathrm{U}=\left\{U_{i}\right\}$ of $Y$ by pre-compact sets and continuous functions $\sigma_{i j}$ from $U_{i j}$ into Aut $K$ with the point-norm topology such that $\sigma=\left\{\sigma_{i j}\right\}$ form a 1 -cocycle in the set $Z^{1}(\mathrm{U}, \mathcal{S})$ (where we'll use $\mathcal{S}$ for the sheaf of germs of continuous Aut $K$ valued functions on $Y$ ) such that $\mathscr{B}$ is isomorphic to

$$
\coprod_{i} U_{i} \times K / \sim
$$

where we identify $(j, u, T)$ with $\left(i, u, \sigma_{i j}(u)(T)\right)$. Furthermore, the Dixmier-Douady class $\delta(\mathscr{B})$ is the image of $\Delta([\sigma]) \in H^{2}(Y, \mathcal{S})$ in $H^{3}(Y ; \mathbb{Z})$ where $\Delta: H^{1}(Y, \mathcal{S}) \rightarrow$ $H^{2}(Y, \delta)$ is the bijection given in [8, Proposition 4.83]. Thus, refining $\mathbf{U}$ if necessary, 
we can assume that there are continuous functions $\mu_{i j}$ from $U_{i j}$ into the unitary group $U(K)$ of (the multiplier algebra of) $K$ with the strict topology such that

$$
\sigma_{i j}(u)=\operatorname{Ad} \mu_{i j}(u) \quad \text { for all } u \in U_{i j} .
$$

(Of course, $U(K)$ is the isomorphic to the unitary group of the underlying Hilbert space of $K$ endowed with the strong topology.) Then $\Delta([\sigma])$ is represented by $v:=\left\{v_{i j k}\right\} \in H^{2}(\mathbf{U}, \mathcal{S})$ given by $\nu_{i j k}(u) \mu_{i k}(u)=\mu_{i j}(u) \mu_{j k}(u)$.

If $\mathscr{B}$ is of the form (8), then $\mathscr{A}:=p^{*} \mathscr{B}$ is of the form

$$
\coprod_{i} p^{-1}\left(U_{i}\right) \times K / \sim
$$

where we identify $(j, \omega, T)$ with $\left(i, \omega, \sigma_{i j}(\dot{\omega})(T)\right)$. Thus if $\left(p^{*} \mathscr{B}, \alpha\right) \in \mathfrak{B r}(G)$, there must be continuous groupoid homomorphisms

$$
\pi_{i}:\left.G\right|_{p^{-1}\left(U_{i}\right)} \rightarrow \text { Aut } K
$$

such that

$$
\alpha\left(\omega, \omega^{\prime}\right)\left(\left[i, \omega^{\prime}, T\right]\right)=\left[i, \omega, \pi_{i}\left(\omega, \omega^{\prime}\right)(T)\right] .
$$

Note that if $\left.\left(\omega, \omega^{\prime}\right) \in G\right|_{p^{-1}\left(U_{i}\right)}$, then $\dot{\omega}=\dot{\omega}^{\prime}$ and

$$
\sigma_{i j}(\dot{\omega}) \circ \pi_{i}\left(\omega, \omega^{\prime}\right)=\pi_{j}\left(\omega, \omega^{\prime}\right) \circ \sigma_{i j}(\dot{\omega}) .
$$

Assume that $G$ is locally trivial with $k_{i}, h_{i}, \xi_{i}$ and $\phi_{i j}$ defined as in Section 1. Then we fix some $z_{i} \in X$ and define

$$
\psi_{i}: p^{-1}\left(U_{i}\right) \rightarrow \text { Aut } K
$$

by

$$
\psi_{i}(\omega)=\pi_{i}\left(k_{i}^{-1}\left(\xi_{i}(\omega), \dot{\omega}, z_{i}\right)\right)
$$

Then, as in Lemma 4,

$$
\pi_{i}\left(\omega, \omega^{\prime}\right)=\psi_{i}(\omega) \psi_{i}\left(\omega^{\prime}\right)^{-1}
$$

Now we observe that

$$
\begin{aligned}
& \psi_{i}(\omega)^{-1} \circ \sigma_{i j}(\dot{\omega}) \circ \psi_{j}(\omega) \\
& \quad=\pi_{i}\left(k_{i}^{-1}\left(\xi_{i}(\omega), \dot{\omega}, a_{i}\right)\right)^{-1} \circ \pi_{i}\left(k_{j}^{-1}\left(\xi_{j}(\omega), \dot{\omega}, z_{j}\right)\right) \circ \sigma_{i j}(\dot{\omega})
\end{aligned}
$$

which, since $\phi_{j i}(\dot{\omega})\left(\xi_{j}(\omega)\right)=\xi_{i}(\omega)$, is

$$
\begin{aligned}
& =\pi_{i}\left(k_{i}^{-1}\left(z_{i}, \dot{\omega}, \xi_{i}(\omega)\right)\right) \circ \pi_{i}\left(k_{i}^{-1}\left(\xi_{i}(\omega), \dot{\omega}, \phi_{j i}(\dot{\omega})\right)\right) \circ \sigma_{i j}(\dot{\omega}) \\
& =\pi_{i}\left(k_{i}^{-1}\left(z_{i}, \dot{\omega}, \phi_{j i}(\dot{\omega})\left(z_{j}\right)\right)\right) \circ \sigma_{i j}(\dot{\omega}) .
\end{aligned}
$$

In particular, (13) depends only on the class $\dot{\omega}$ of $\omega$ in $Y$. 
THEOREM 8. Suppose that $G$ is a second countable, locally compact, locally trivial, proper principal groupoid, that $\mathscr{B}$ is a locally trivial $K$-bundle over $Y$ and $\left(p^{*} \mathscr{B}, \alpha\right) \in$ $\mathfrak{B r}(G), \mathbf{U}, \psi_{i}$ and $\sigma_{i j}$ are as defined above. Then

$$
\beta_{i j}(\dot{\omega})=\psi_{i}(\omega)^{-1} \circ \sigma_{i j}(\dot{\omega}) \circ \psi_{j}(\omega)
$$

defines a class $\beta$ in $H^{1}(Y, \mathcal{S})$ which depends only on $\left[p^{*} \mathscr{B}, \alpha\right] \in \operatorname{Br}(G)$. Furthermore, the Dixmier-Douady class of the corresponding groupoid crossed product $C^{*}\left(G ; p^{*} \mathscr{B}\right)$ is the image of $\Delta(\beta)$ in $H^{3}(Y ; \mathbb{Z})$.

PROOF. It follows from Theorem 6 that $\delta\left(C^{*}\left(G ; p^{*} \mathscr{B}\right)\right)$ depends only on $\left[p^{*} \mathscr{B}, \alpha\right]$. It is clear from (14) and (15) that $\left\{\beta_{i j}\right\}$ defines a cocycle in $Z^{1}(\mathrm{U}, \delta)$ and therefore a class $\beta$ in $H^{1}(Y, \delta)$. It will suffice to see that the image of $\Delta(\beta)$ is $\delta\left(C^{*}\left(G ; p^{*} \mathscr{B}\right)\right)$.

Since the proof of $\left[3\right.$, Theorem 1] implies that $C^{*}\left(G, p^{*} \mathscr{B}\right)$ is Morita equivalent to $C_{0}\left(Y, p^{*} \mathscr{B} / G\right)$ where $p^{*} \mathscr{B} / G$ is the orbit space on $p^{*} \mathscr{B}$ with respect to the right action of $G$ given by $[i, \omega, T] \cdot\left(\omega, \omega^{\prime}\right)=\left[i, \omega^{\prime}, \pi_{i}\left(\omega^{\prime}, \omega\right)(T)\right]$, it will suffice to compute $\delta\left(p^{*} \mathscr{B} / G\right)$. We'll denote the image of $[i, \omega, T]$ in $p^{*} \mathscr{B} / G$ by $\llbracket i, \omega, T \rrbracket$. Let $\mathrm{V}=\left\{V_{i}\right\}$ be a cover of $Y$ such that $\bar{V}_{i} \subset U_{i}$ ([8, Lemma 4.32]). Let $P$ be a rank-one projection in $K$. If $w \in p^{-1}\left(U_{i}\right)$, then

$$
\left[i, \omega, \psi_{i}(\omega)(T)\right] \cdot\left(\omega, \omega^{\prime}\right)=\left[i, \omega^{\prime}, \pi_{i}\left(\omega^{\prime}, \omega\right) \circ \psi_{i}(\omega)(T)\right]=\left[i, \omega^{\prime}, \psi_{i}\left(\omega^{\prime}\right)(T)\right] .
$$

Therefore $\dot{\omega} \mapsto \llbracket i, \omega, \psi_{i}(\omega)(P) \rrbracket$ is a rank-one projection field in $\left.\left(p^{*} \mathscr{B} / G\right)\right|_{p^{-1}\left(U_{i}\right)}$. Fell's vector-valued Tietze extension theorem [2, Theorem II.14.8] implies that the restriction to $\bar{V}_{i}$ extends to a global section $q_{i} \in C_{0}\left(Y, p^{*} \mathscr{B} / G\right)$ such that

$$
q_{i}(\dot{\omega})=\llbracket i, \omega, \psi_{i}(\omega)(P) \rrbracket \quad \text { for all } \dot{\omega} \in V_{i} .
$$

We may as well assume that there are continuous functions $\theta_{i j}: U_{i j} \rightarrow U(K)$ such that

$$
\beta_{i j}(\dot{\omega})=\operatorname{Ad} \theta_{i j}(\dot{\omega}) \text { for all } \dot{\omega} \in U_{i j}
$$

Of course, $\mathbf{V}$ is a refinement of $\mathbf{U}$ with the same index set and $\Delta(\beta)$ is given by the 2-cocycle $\left\{\epsilon_{i j k}\right\}$ on $\mathbf{V}$ defined by

$$
\theta_{i j}(\dot{\omega}) \theta_{j k}(\dot{\omega})=\epsilon_{i j k}(\dot{\omega}) \theta_{i k}(\dot{\omega}) \quad \text { for } \dot{\omega} \in V_{i j k}
$$

As above, there is a $v_{i j} \in C_{0}\left(Y, p^{*} \mathscr{B} / G\right)$ such that

$$
v_{i j}(\dot{\omega})=\llbracket i, \omega, \psi_{i}(\omega)\left(P \theta_{i j}(\dot{\omega})^{*}\right) \rrbracket \quad \text { for } \dot{\omega} \in \overline{V_{i j}} .
$$


For $\dot{\omega} \in V_{i j}$, we certainly have

$$
v_{i j}(\dot{\omega}) v_{i j}(\dot{\omega})^{*}=\llbracket i, \omega, \psi_{i}(\omega)(P) \rrbracket=q_{i}(\dot{\omega}),
$$

while

$$
\begin{aligned}
v_{i j}(\dot{\omega})^{*} v_{i j}(\dot{\omega}) & =\llbracket i, \omega, \psi_{i}(\omega)\left(\theta_{i j}(\dot{\omega}) P \theta_{i j}(\dot{\omega})^{*}\right) \rrbracket \\
& =\llbracket i, \omega, \psi_{i}(\omega) \circ \beta_{i j}(\dot{\omega})(P) \rrbracket \\
& =\llbracket i, \omega, \sigma_{i j}(\dot{\omega}) \circ \psi_{j}(\omega)(P) \rrbracket \\
& =\llbracket j, \omega, \psi_{j}(\omega)(P) \rrbracket \\
& =q_{j}(\dot{\omega}) .
\end{aligned}
$$

Thus we have just the set-up for [8, Lemma 5.28]. So to find the Dixmier-Douady class, we compute that

$$
\begin{aligned}
v_{j k}(\dot{\omega})^{*} v_{i j}(\dot{\omega})^{*} & =\llbracket j, \omega, \psi_{j}(\omega)\left(\theta_{j k}(\dot{\omega}) P\right) \rrbracket \llbracket i, \omega, \psi_{i}(\omega)\left(\theta_{i j}(\dot{\omega}) P\right) \rrbracket \\
& =\llbracket i, \omega, \sigma_{i j}(\dot{\omega}) \circ \psi_{j}(\omega)\left(\theta_{j k}(\dot{\omega}) P\right) \rrbracket \llbracket i, \omega, \psi_{i}(\omega)\left(\theta_{i j}(\dot{\omega}) P\right) \rrbracket \\
& =\llbracket i, \omega, \psi_{i}(\omega) \circ \beta_{i j}(\dot{\omega})\left(\theta_{j k}(\dot{\omega}) P\right) \rrbracket \llbracket i, \omega, \psi_{i}(\omega)\left(\theta_{i j}(\dot{\omega}) P\right) \rrbracket \\
& =\llbracket i, \omega, \psi_{i}(\omega)\left(\theta_{i j}(\dot{\omega}) \theta_{j k}(\dot{\omega}) P \theta_{i j}(\dot{\omega})^{*}\right) \rrbracket \llbracket i, \omega, \psi_{i}(\omega)\left(\theta_{i j}(\dot{\omega}) P\right) \rrbracket \\
& =\epsilon_{i j k}(\dot{\omega}) \llbracket i, \omega, \psi_{i}(\omega)\left(\theta_{i k}(\dot{\omega}) P\right) \rrbracket \\
& =\epsilon_{i j k}(\dot{\omega}) v_{i k}(\dot{\omega})^{*} .
\end{aligned}
$$

Thus $\left[8\right.$, Lemma 5.28] implies that $\Delta(\beta)=\left[\left\{\epsilon_{i j k}\right\}\right]$ gives the Dixmier-Douady class of $C^{*}\left(G ; p^{*} \mathscr{B}\right)$ as claimed.

DEFINITION 9. Let $G=\Omega{ }_{p}^{*} \Omega$ be a locally trivial proper principal groupoid, and let $\mathscr{B}$ be a locally trivial $K$-bundle with structure group Aut $K$. Then an action of $\alpha$ of $G$ on $p^{*} \mathscr{B}$ is called a locally unitary pull-back if there is a cover $\mathbf{U}$ of $Y$ and continuous functions $v_{i}: p^{-1}\left(U_{i}\right) \rightarrow U(K)$ such that $\psi_{i}(\omega)=\operatorname{Ad} v_{i}(\omega)$ for all $\omega \in p^{-1}\left(U_{i}\right)$.

REMARK 10. Note that if $\alpha$ is as in Definition 9, then there are actually groupoid homomorphisms $u_{i}:\left.G\right|_{p^{-1}\left(U_{i}\right)} \rightarrow U(K)$ such that $\pi_{i}\left(\omega, \omega^{\prime}\right)=\operatorname{Ad} u_{i}\left(\omega, \omega^{\prime}\right)$, where $\boldsymbol{u}_{i}\left(\omega, \omega^{\prime}\right)=\psi_{i}(\omega) \psi_{i}\left(\omega^{\prime}\right)^{-1}$.

Since $\sigma_{i j}(\dot{\omega}) \circ \pi_{i}\left(\omega, \omega^{\prime}\right)=\pi_{j}\left(\omega, \omega^{\prime}\right) \circ \sigma_{i j}(\dot{\omega})$, there are continuous groupoid homomorphisms

$$
\lambda_{i j}:\left.G\right|_{p^{-1}\left(U_{i}\right)} \rightarrow \mathbb{C} \text { such that } \sigma_{i j}(\dot{\omega})^{-}\left(u_{j}\left(\omega, \omega^{\prime}\right)\right)=\lambda_{i j}\left(\omega, \omega^{\prime}\right) u_{j}\left(\omega, \omega^{\prime}\right) .
$$

REMARK 11. We want to think of $\left\{\lambda_{i j}\right\}$ as a generalized Phillips-Raeburn obstruction for $\alpha$. It is possible to view (18) as defining a class in a certain sheaf cohomology group, which we would naturally denote $H^{1}(Y, \mathcal{G})$, but we will not pursue that here. 
EXAMPLE 12. Suppose that $\mathscr{B}$ is a locally trivial $K$-bundle with transition functions $\left\{\sigma_{i j}\right\}$ as above and that $\gamma: H \rightarrow$ Aut $C_{0}(Y, \mathscr{B})$ is a locally unitary automorphism group. If $p: \Omega \rightarrow Y$ is a principal $H$-bundle, then let $G=\Omega{ }_{p}^{*} \Omega$ and $\alpha$ the action of $G$ on $p^{*} \mathscr{B}$ obtained by the pull-back: $p^{*} \gamma: H \rightarrow$ Aut $p^{*} \mathscr{B}$ given by $p^{*} \gamma_{s}(f)(\omega)=\gamma_{s}\left(f\left(s^{-1} \cdot \omega\right)\right)$. Since $\gamma$ is locally unitary, we can assume $\mathbf{U}$ has been taken so that there are strongly continuous homomorphisms $v^{i}: U_{i} \times H \rightarrow$ $U(K)$ such that $\alpha(s \cdot \omega, \omega)([i, \dot{\omega}, T])=\left[i, \dot{\omega}, v_{s}^{i}(\dot{\omega}) T v_{s}^{i}(\dot{\omega})^{*}\right]$. Then the continuous functions $\zeta_{i j}: U_{i j} \rightarrow \widehat{H}$ defined by $\sigma_{i j}(\dot{\omega})\left(v_{s}^{j}(\dot{\omega})\right)=\zeta_{i j}(\dot{\omega})(s) v_{s}^{i}(\dot{\omega})$ determine a class $\zeta(\gamma) \in H^{1}\left(Y, \widehat{\mathcal{H}}_{\mathrm{ab}}\right)$ called the Phillips-Raeburn obstruction for $\gamma$ (see, for example, [1, Proposition 3.3]). (Here $\widehat{\mathcal{H}}_{\mathrm{ab}}$ is the dual of the abelianization $H / \overline{[H, H]}$ of $H$ which coincides with the collection of characters on $H$.) Note that for $\alpha$ as above,

$$
\pi_{i}\left(\omega, \omega^{\prime}\right)=\operatorname{Ad} u_{i}\left(\omega, \omega^{\prime}\right) \quad \text { where } \quad u_{i}(s \cdot \omega, \omega)=v_{s}^{i}(\dot{\omega})
$$

Thus the generalized Phillips-Raeburn obstruction and the original are, in this case, related by $\lambda_{i j}(s \cdot \omega, \omega)=\zeta_{i j}(\dot{\omega})(s)$.

THEOREM 13. Suppose that $G$ is a second countable, locally compact, locally trivial, proper principal groupoid, and that $\left(p^{*} \mathscr{B}, \alpha\right) \in \mathfrak{B r}(G)$ is a locally unitary pull-back, with $\mathscr{B}$ a locally trivial $K$-bundle as above. Then, with $\lambda_{i j}$ as defined in (18), the equation

$$
\tau_{i j k}(\dot{\omega})=\lambda_{i j}\left(k_{j}^{-1}\left(z_{j}, \dot{\omega}, \phi_{k j}(\dot{\omega})\left(z_{k}\right)\right)\right)
$$

defines an element $\langle\alpha, p\rangle$ in $H^{3}(Y ; \mathbb{Z})$ which depends only on $\left[p^{*} \mathscr{B}, \alpha\right] \in \operatorname{Br}(G)$ and $\delta(\mathscr{B})$. Furthermore, the Dixmier-Douady class of the crossed-product is given by the formula

$$
\delta\left(C^{*}\left(G, p^{*} \mathscr{B}\right)\right)=\delta(\mathscr{B})+\langle\alpha, p\rangle
$$

PROOF. Since the class of the crossed product only depends on $\left[p^{*} \mathscr{B}, \alpha\right]$, it will suffice to establish (19). We adopt the notation used in the proof of Theorem 8 . Thus $\delta\left(C^{*}\left(G ; p^{*} \mathscr{B}\right)\right)$ is given by $\left\{\epsilon_{i j k}\right\}$ where

$$
\begin{aligned}
v_{i k}(\dot{\omega})^{*} v_{i j}(\dot{\omega})^{*} & =\epsilon_{i j k}(\dot{\omega}) v_{i k}(\dot{\omega})^{*} \quad \text { for all } \dot{\omega} \in V_{i j k}, \quad \text { and } \\
v_{i j}(\dot{\omega}) & :=\llbracket i, \omega, \psi_{i}(\omega)(P) \rrbracket .
\end{aligned}
$$

We may assume that $\delta(\mathscr{B})$ is given by a cocycle $\left\{\nu_{i j k}\right\}$ which is determined by functions $\mu_{i j}: U_{i j} \rightarrow U(K)$ such that

$$
\sigma_{i j}(\dot{\omega})=\operatorname{Ad} \mu_{i j}(\dot{\omega}) \quad \text { and } \quad v_{i j k}(\dot{\omega}) \mu_{i k}(\dot{\omega})=\mu_{i j}(\dot{\omega}) \mu_{j k}(\dot{\omega})
$$


Since $\alpha$ is a locally unitary pull-back, we can, in view of (14), write

$$
\beta_{i j}(\dot{\omega})=\operatorname{Ad} \theta_{i j}(\dot{\omega}), \quad \text { where } \quad \theta_{i j}(\dot{\omega})=u_{i}\left(k_{i}^{-1}\left(z_{i}, \dot{\omega}, \phi_{j i}(\dot{\omega})\left(z_{j}\right)\right)\right) \mu_{i j}(\dot{\omega})
$$

with $u_{i}$ as defined in Remark 10. But then

$$
\begin{aligned}
\theta_{i j}(\dot{\omega}) \theta_{j k}(\dot{\omega})= & u_{i}\left(k_{i}^{-1}\left(z_{i}, \dot{\omega}, \phi_{j i}(\dot{\omega})\left(z_{j}\right)\right)\right) \mu_{i j}(\dot{\omega}) \\
& \times u_{j}\left(k_{j}^{-1}\left(z_{j}, \dot{\omega}, \phi_{k j}(\dot{\omega})\left(z_{k}\right)\right)\right) \mu_{j k}(\dot{\omega}) \\
= & \tau_{i j k}(\dot{\omega}) u_{i}\left(k_{i}^{-1}\left(z_{i}, \dot{\omega}, \phi_{j i}(\dot{\omega})\left(z_{j}\right)\right)\right) u_{i}\left(k_{j}^{-1}\left(z_{j}, \dot{\omega}, \phi_{k j}(\dot{\omega})\left(z_{k}\right)\right)\right) \\
& \times \mu_{i j}(\dot{\omega}) \mu_{j k}(\dot{\omega}) \\
= & \tau_{i j k}(\dot{\omega}) \nu_{i j k}(\dot{\omega}) u_{i}\left(k_{i}^{-1}\left(z_{i}, \dot{\omega}, \phi_{k i}(\dot{\omega})\left(z_{k}\right)\right)\right) \mu_{i k}(\dot{\omega}) . \\
= & \tau_{i j k}(\dot{\omega}) v_{i j k}(\dot{\omega}) \theta_{i k}(\dot{\omega}) .
\end{aligned}
$$

It follows that $\left\{\tau_{i j k} \nu_{i j k}\right\}$ is a 2-cocycle defining $\delta\left(C^{*}\left(G, p^{*} \mathscr{B}\right)\right)$. In particular, $\tau_{i j k}$ is also a 2-cocycle and we're done.

EXAMPLE 14. We should compare our Theorem 13, in the setting of Example 12, with [6, Theorem 1.5]. To see that our formula coincides with Raeburn and Rosenberg's, we have to see that our $\langle\alpha, p\rangle$ is the same as their $\langle\gamma, p\rangle$. However,

$$
\lambda_{i j}\left(k_{j}^{-1}\left(z_{j}, \dot{\omega}, \phi_{k j}(\dot{\omega})\left(z_{k}\right)\right)\right)=\lambda_{i j}\left(h_{j}^{-1}\left(z_{j}, \dot{\omega}\right), h_{j}^{-1}\left(z_{k} \cdot s_{k j}(\dot{\omega}), \dot{\omega}\right)\right) .
$$

If, as we may, we take $z_{i}=e$ for all $i$, then

$$
\lambda_{i j}\left(\omega, s_{k j}(\dot{\omega}) \cdot \omega\right)=\zeta_{i j}(\dot{\omega})\left(s_{j k}(\dot{\omega})\right)
$$

which certainly appears to be a representative for Raeburn and Rosenberg's $\langle\gamma, p\rangle$. Unfortunately we used a different convention in (2) and (4) from the one used in [6, Theorem 1.5]. In view of this, our $\langle\alpha, p\rangle$ should be $-\langle\gamma, p\rangle$. The difference seems to be that the expression " $\operatorname{Ad}\left(\boldsymbol{u}_{\lambda_{i j}(t)}^{i}(t) v_{i j}(t)\right)$ " in the middle of page 15 in the proof of $\left[6\right.$, Theorem 1.5] should be "Ad $\left(u_{\lambda_{j}(t)}^{i}(t) v_{i j}(t)\right)$ ".

\section{References}

[1] S. Echterhoff and D. P. Williams, 'Locally inner actions on $C_{0}(X)$-algebras', J. Operator Theory 45 (2001), 131-160.

[2] J. M. G. Fell and R. Doran, Representations of *-algebras, locally compact groups, and Banach *-algebraic bundles, Vol. I \& II (Academic Press, New York, 1988).

[3] I. Fulman, P. S. Muhly and D. P. Williams, 'Continuous-trace groupoid crossed products', Proc. Amer. Math. Soc. 132 (2004), 707-717. 
[4] A. Kumjian, P. S. Muhly, J. N. Renault and D. P. Williams, 'The Brauer group of a locally compact groupoid', Amer. J. Math. 120 (1998), 901-954.

[5] P. S. Muhly, J. Renault and D. P. Williams, 'Equivalence and isomorphism for groupoid $C^{*}$-algebras', J. Operator Theory 17 (1987), 3-22.

[6] I. Raeburn and J. Rosenberg, 'Crossed products of continuous-trace $C^{*}$-algebras by smooth actions', Trans. Amer. Math. Soc. 305 (1988), 1-45.

[7] I. Raeburn and D. P. Williams, 'Pull-backs of $C^{*}$-algebras and crossed products by certain diagonal actions', Trans. Amer. Math. Soc. 287 (1985), 755-777.

[8] - Morita equivalence and continuous-trace $C^{*}$-algebras, Mathematical Surveys and Monographs 60 (American Mathematical Society, Providence, RI, 1998).

Department of Mathematics

University of Iowa

Iowa City, IA 52242

USA

e-mail: pmuhly@math.uiowa.edu
Department of Mathematics

Dartmouth College Hanover, NH 03755-3551

USA

e-mail: dana.williams@dartmouth.edu 